\title{
HOLOMORPHIC FUNCTIONS WITH SPIRAL ASYMPTOTIC PATHS
}

\author{
W. SEIDEL *
}

1. Let $f(z)$ be a holomorphic and unbounded function in $|z|<1$, with the property that it remains bounded on some spiral $S$ in $|z|<1$ which approaches $|z|=1$ asymptotically. The existence of such functions was first established by G. Valiron." Accordingly, we shall refer to such functions as functions of class $(V)$ relative to $S$. More recently, F. Bagemihl and W. Seidel obtained examples of functions holomorphic and unbounded in $|z|<1$ which approach prescribed finite or infinite values as $|z| \rightarrow 1$ on any given enumerable set of disjunct spirals which approach $|z|=1$ asymptotically, ${ }^{2)}$ as well as on certain sets of such spirals having the power of the continuum. ${ }^{3}$

In his 1936 paper, Valiron established various properties of functions of class $(V)$ relative to a spiral $S$, of which we mention, for future reference, the following:

I. If $f(z)$ is of class $(V)$ relative to a spiral $S$, there exists a spiral path on which $f(z)$ tends to infinity.

In this paper, we continue the study of functions with spiral asymptotic paths, and shall derive some further properties of such functions.

2. In the sequel, we shall use the term "spiral" in the following sense. Let $\zeta(t)$ be a continuous, complex-valued function for $0 \leqq t<\infty$ with the properties:

Received August 2, 1958

* This paper was written under the sponsorship of the National Science Foundation.

1) G. Valiron. 1. Sur certaines singularites des fonctions holomorphes dans un cercle, Comptes Rendus de l'Académie des Sciences de Paris, vol. 198 (1934), pp. 2065-2067, and 2. Sur les singularités de certaines fonctions holomorphes et de leurs inverses, Journal de Mathématiques pures et appliquées (9), vol. 15 (1936), pp. 423-435.

2) F. Bagemihl and W. Seidel. Spiral and other asymptotic paths, and paths of complete indetermination, of analytic and meromorphic functions, Proceedings of the National Academy of Sciences, vol. 39 (1953), pp. 1251-1258.

3) F. Bagemihl and W. Seidel. Some boundary properties of analytic functions, Mathematische Zeitschrift, vol. 61 (1954), pp. 186-199. 


$$
0<|\zeta(t)|<1, \lim _{t \rightarrow \infty}|\zeta(t)|=1, \lim _{t \rightarrow \infty} \arg \zeta(t)=\infty,
$$

and let the equation $z=\zeta(t)$ define a simple curve. For any value of $t$, starting with the point $\zeta(t)$ on the curve, describe the curve in the sense of increasing $t$ and let $t^{\prime}$ denote the first value of $t$ for which $\arg \zeta\left(t^{\prime}\right)=\arg \zeta(t)+$ $2 \pi$. We shall denote by $\rho\left(\zeta(t), \zeta\left(t^{\prime}\right)\right)$ the non-Euclidean ${ }^{4)}$ (hyperbolic) distance between these two points:

$$
\rho\left(\zeta(t), \zeta\left(t^{\prime}\right)\right)=\frac{1}{2} \log \frac{1+u}{1-u}, u=\left|\frac{\zeta(t)-\zeta\left(t^{\prime}\right)}{1-\overline{\zeta(t)} \zeta\left(t^{\prime}\right)}\right| .
$$

We shall also find it convenient to introduce the following measures for the "tightness" of a spiral $S$ whose equation is $z=\zeta(t)$ :

$$
\mu(S)=\lim _{t \rightarrow \infty} \rho\left(\zeta(t), \zeta\left(t^{\prime}\right)\right), \bar{\mu}(S)=\varlimsup_{t \rightarrow \infty} \rho\left(\zeta(t), \zeta\left(t^{\prime}\right)\right) .
$$

In order to simplify the formulation of theorems, we shall also introduce certain terms and notations which have become familiar in recent years."

(a) Given a point $\tau$ on $|z|=1$, the set of all points $z$ in $|z|<1$ for which

$$
|\arg (1-\bar{\tau} z)| \leqq \beta,|z-\tau|<\varepsilon,
$$

where $0<\beta<\frac{\pi}{2}$ and $\varepsilon$ is chosen so small that the boundary of the resulting set has only the point $\tau$ in common with $|z|=1$, shall be called a symmetric Stolz angle with vertex $\tau$ and of opening $2 \beta$, and will be denoted by $\Delta_{\tau, \beta}$.

(b) Let $\Sigma$ be any subset of $|z|<1$, whose closure has at least one point in common with $|z|=1$. By the range of a function $f(z)$ in $\Sigma, R(f, \Sigma)$, we shall mean the set of all complex values $a$ for which there exists a sequence of points $\left\{z_{n}\right\}$, with $z_{n} \in \Sigma, \lim _{n \rightarrow \infty}\left|z_{n}\right|=1$, such that $f\left(z_{n}\right)=a$.

(c) Let $G$ be the set of all finite complex numbers and $A$ a subset of $G$. We shall denote by $C A$ the complement of $A$, relative to $G$.

3. TheOREM 1. Let $f(z)$ be of class $(V)$ relative to a spiral $S$, for which

4) In the sequel, we shall abbreviate the expression "non-Euclidean" to $n-E$. For the facts concerning $n-E$ geometry which we shall employ in this paper, see, for example, C. Carathéodory. Conformal Representation, second edition, Cambridge, University Press, 1952, Chapter II.

5) See, for example, E. F. Collingwood and M. L. Cartwright. Boundary theorems for a function meromorphic in the unit circle, Acta Mathematica, vol. 87 (1952), pp. 83-146. 


$$
\mu(S)<\infty .
$$

Then; there exists a spiral $S^{\prime}$ in $|z|<1$, such that, denoting by $\Sigma$ the union of all $n-E$ open circular discs with $n-E$ centers on $S^{\prime}$ and of fixed $n-E$ radius, the set $C R(f, \Sigma)$ consists of at most one point.

Proof. Let $S$ be given by $\zeta(t), 0 \leqq t<\infty$. In view of condition (4), there exists an increasing sequence $\left\{t_{n}\right\}$, with $\lim _{n \rightarrow \infty} t_{n}=\infty$, and a positive constant $M$ such that

$$
\rho\left(\zeta\left(t_{n}\right), \zeta\left(t_{n}^{\prime}\right)\right)<M, n=1,2, \ldots
$$

Consider the sequence of functions

$$
g_{n}(z)=f\left(\frac{z+\zeta\left(t_{n}\right)}{1+\zeta\left(t_{n}\right) z}\right)
$$

each of which is clearly holomorphic in $|z|<1$.

Since $f(z)$ is of class $(V)$ relative to $S$, there exists a positive constant $B$ such that $\left|f\left(\zeta\left(t_{n}\right)\right)\right|<B$ for $n=1,2, \ldots$ Hence, by (6), we have

$$
\left|g_{n}(0)\right|<B
$$

The family $\left\{g_{n}(z)\right\}$ can not be normal in $|z|<1$. For, if it were, no subsequence could tend to infinity, because of (7). Hence, the sequence $\{n\}$ of natural numbers would contain a subsequence $\left\{n_{k}\right\}$ for which $\left\{g_{n_{k}}(z)\right\}$ would tend uniformly in every closed circular disc $|z| \leqq \lambda<1$ to some function $g(z)$ holomorphic in $|z|<1$.

Now, the spiral $S$ satisfies condition (5). Consider any circle $|z| \leqq d<1$ whose $n-E$ radius $\frac{1}{2} \log \frac{1+d}{1-d}$ is greater than M. Since $g(z)$ is bounded in $|z| \leqq d$, there exists a positive constant $L$ such that $\left|g_{n_{k}}(z)\right|<L$ for $|z| \leqq d$ and for all values of $k$. But the set of values which $g_{l_{k}}(z)$ assumes in $|z|<d$ is the same as the set of values which $f(z)$ assumes in the $n-E$ disc $D_{k, d}$ whose $n-E$ center is $\zeta\left(t_{n_{k}}\right)$ and whose $n-E$ radius is $\frac{1}{2} \log \frac{1+d}{1-d}$. By the above choice of $d$, it is clear that the disc $D_{k, d}$ contains the point $\zeta\left(t_{n_{k}}^{\prime}\right)$ and also $|f(z)|<L$ in $D_{k, d}$. This, however, is impossible, since, according to $I, f(z)$ must possess a spiral path $P$ on which it tends to infinity, and this path would have to cross the circles $D_{k, d}$ infinitely many times.

Since the family $\left\{g_{n}(z)\right\}$ can not be normal, there exists a point $z_{0}$, with 
' $z_{0} \mid<1$, such that in every neighborhood of $z_{0}$ every value, except at most one, is assumed by infinitely many functions of the family. If it can be shown that

$$
z(t)=\frac{z_{0}+\zeta(t)}{1+\zeta(\bar{t}) z_{0}}
$$

is a spiral in the sense of $\S 2$, the conclusion of the theorem will follow at once, with $S^{\prime}$ defined by (8).

We have $0<|z(t)|<1$, except possibly for one value of $t$. Moreover, (1) implies that $\lim _{t \rightarrow \infty}|z(t)|=1$. Furthermore, we have $z(t)=\zeta(t)$ if $z_{0}=0$, and if $0<\left|z_{0}\right|<1$, we have

$$
\arg z(t)=\arg \left(z_{0}+\zeta(t)\right)-\arg z_{0}-\arg \left(\frac{1}{z_{0}}+\zeta(t)\right) .
$$

Here the first term on the right tends to infinity as $t \rightarrow \infty$, while the two remaining terms stay bounded. Finally, the equation $z=z(t)$ defines a simple curve. For, if not, there would be two distinct values $t_{1}, t_{2}$ for which $z\left(t_{1}\right)=$ $z\left(t_{2}\right)$. But (8) implies the relation

$$
\zeta(t)=\frac{z(t)\left(1-\left|z_{0}\right|^{2}\right)-z_{0}\left(1-|z(t)|^{2}\right)}{1-\left|z_{0}\right|^{2}|z(t)|^{2}},
$$

so that we would obtain $\zeta\left(t_{1}\right)=\zeta\left(t_{2}\right)$, which is impossible. This completes the proof.

It is evident that Theorem 1 is a kind of analogue of Julia's Theorem for entire functions.

Remark. That in Theorem $1, C R(f, \Sigma)$ may not be empty is evident from the fact that if $f(z)$ is of class $(V)$ relative to a spiral, so is the function $e^{f(z)}$.

4. TheOREM 2. Let $f(z)$ be of class $(V)$ relative to a spiral $S$, for which

$$
\bar{\mu}(S)<\infty,
$$

and let $\tau$ be an arbitrary point of $|z|=1$. Then $C R\left(f, \Delta_{-, \alpha}\right)$ consists of at most one point for every Stolz angle $\Delta:, \alpha$, for which

$$
\bar{\mu}(S)<\frac{1}{2} \log \cot \left(\frac{\pi}{4}-\frac{\alpha}{2}\right) .
$$

Proof. Let $z_{1}=\zeta\left(t_{1}\right)$ be any point of intersection of $S$ with the radius terminating in the point - . We define $z_{n}=\zeta\left(t_{n}\right)$ inductively by the relation 
$z_{n+1}=\zeta\left(t_{n}^{\prime}\right)$ in the notation of $\S 1$. Clearly, $\lim _{n \rightarrow \infty}\left|z_{n}\right|=1$. Form the functions

$$
g_{n}(z)=f\left(\begin{array}{c}
z+z_{n} \\
1+\bar{z}_{n} z
\end{array}\right), n=1,2, \ldots
$$

which are evidently holomorphic in $|z|<1$ and let the positive number $q$ satisfy the inequalities

$$
\bar{\mu}(S)<q<\frac{1}{2} \log \cot \left(\frac{\pi}{4}-\frac{\alpha}{2}\right) .
$$

It follows by exactly the same argument as in the proof of Theorem 1 that the family $\left\{g_{n}(z)\right\}$ is not normal in the $n-E$ disc whose $n-E$ center is 0 and whose $n-E$ radius is $q$, i.e. in the Euclidean circular disc $|z|<\frac{e^{2 q}-1}{e^{2 q}+1}$.

Thus, there exists a point $z_{0}$, with $\left|z_{0}\right|<\frac{e^{2 q}-1}{e^{2 q}+1}$ such that every value, except perhaps one, is assumed by infinitely many of the functions of the family $\left\{g_{n}(z)\right\}$ in every $n-E$ disc with $n-E$ center $z_{0}$. Choose the $n-E$ radius of such a disc so small that the disc lies wholly within the disc $|z|<\frac{e^{2 q}-1}{e^{2 q}+1}$.

Now, $g_{n}(z)$ assumes in this $n-E$ disc the same values as $f(z)$ assumes in the $n-E$ disc of $n-E$ center $z_{n}$ and the same $n-E$ radius. An elementary calculation shows that the union of all $n-E$ circular discs with $n-E$ centers at the points of the radius terminating in - and $n-E$ radius $q$ will be contained in the region $H_{\tau}, \alpha$ bounded by two hypercycles symmetric in the diameter connecting the points $\tau$ and $-\tau$ and forming at $\tau$ angles $\alpha$ and $-\alpha$ with the diameter, where $\alpha$ is subject to the inequality (12). But in a neighborhood of $\tau$ the region $H_{\tau, \alpha}$ is contained within the Stolz angle $\Delta_{\tau, \alpha}$. This completes the proof.

CoRollary 1. Let $f(z)$ be of class $(V)$ relative to a spiral $S$ for which $\bar{\mu}(S)=0$ and let $\tau$ be an arbitrary point of $|z|=1$. Then $C R\left(f, \Delta_{\tau, \alpha}\right)$ consists of at most one point for every Stolz angle $\Delta_{\tau, \alpha}, \alpha>0$.

Indeed, in this case the positive angle $\alpha$ in the inequality (10) may be taken arbitrarily small.

5. We now turn to the case that a holomorphic function tends to infinity along a spiral path.

THEOREM 3. Let $f(z)$ be holomorphic in $|z|<1$ and let $S: z=\zeta(t), 0 \leqq t<\infty$, 
be a spiral for which

$$
\bar{\mu}(S)<\infty
$$

and

$$
\lim _{t \rightarrow \infty}|f(\zeta(t))|=\infty .
$$

Then there exists a spiral $S^{\prime}$ in $|z|<1$, such that, denoting by $\Sigma$ the union of all $n-E$ open circular discs with $n-E$ centers on $S^{\prime}$ and of fixed $n-E$ radius, the set $C R(f, \Sigma)$ consists of at most one point.

Proof. We form the family of holomorphic functions in $|z|<1$ :

$$
g_{t}(z)=f\left(\frac{z+\zeta(t)}{1+\zeta(t) z}\right), 0 \leqq t<\infty .
$$

Since $g_{t}(0)=f(\zeta(t))$, we have, according to (14),

$$
\lim _{t \rightarrow \infty}\left|g_{t}(0)\right|=\infty
$$

This family can not be normal in $|z|<1$. Indeed, (13) implies the existence of a positive constant $M$ such that

$$
\rho\left(\zeta(t), \zeta\left(t^{\prime}\right)\right)<M
$$

for all sufficiently large values of $t$. Consider any circle $|z| \leqq d$ whose $n-E$ radius $\frac{1}{2} \log \frac{1+d}{1-d}$ is greater than $M$. If $\left\{g_{t}(z)\right\}$ were normal in $|z|<1$, being given any sequence $t_{n}$ of positive numbers for which $t_{n} \rightarrow \infty$, we could extract from the sequence $\left\{g_{t_{n}}(z)\right\}$ a subsequence which, because of (15), would have to tend uniformly to infinity within $|z| \leqq d$. As will be shown, this implies that $\lim _{t \rightarrow \infty}\left|g_{t}(z)\right|=\infty$ uniformly in $|z| \leqq d$.

Indeed, suppose this were not the case. Then there would exist a positive number $M_{0}$, a sequence of values $t_{n}$, with $t_{n} \rightarrow \infty$, and a sequence of points $z_{n}$, with $\left|z_{n}\right| \leqq d$, such that

$$
\left|g_{t_{n}}\left(z_{n}\right)\right| \leqq M_{0}
$$

for $n=1,2,3, \ldots$ But if we apply to this sequence $\left\{g_{t_{n}}(z)\right\}$ the argument of the preceding paragraph, we immediately arrive at a contradiction.

Thus, $\lim _{t \rightarrow \infty}\left|g_{\ell}(z)\right|=\infty$ uniformly in $|z| \leqq d$. 'This implies that $|f(z)|$ tends 
uniformly to infinity within the union of all closed $n-E$ circular discs $D_{t}$ with $n-E$ centers $\zeta(t)$ and $n-E$ radius $\frac{1}{2} \log \frac{1+d}{1-d}$. It follows that, given any positive number $G$, there exists a positive number $\nu$ such that

$$
|f(z)|>G, \quad z \in \bigcup_{t} D_{t}
$$

Since we supposed that

$$
\frac{1}{2} \log \frac{1+d}{1-d}>M
$$

the set $\bigcup_{t>v} D_{t}$ must contain some circular ring $0<\sigma \leqq|z|<1$. This, however, implies that $\lim _{|:| \rightarrow 1}|f(z)|=\infty$ uniformly in $|z|<1$ and, as is well-known, this is impossible.

Thus, we have arrived at a contradiction, which means that the family $\left\{g_{t}(z)\right\}$ can not be normal in $|z|<1$; in fact, it is not even normal in $|z|<d$. Since, on the other hand, every subfamily $\left\{g_{t}(z), 0 \leqq t \leqq A<\infty\right\}$ is normal in $|z|<d$ since it is a bounded family, the failure of the family $\left\{g_{t}(z)\right\}$ to be normal must be due to the presence in it of sequences $\left\{g_{t_{1}}(z)\right\}$, with $t_{n} \rightarrow \infty$, which themselves are not normal. Once this is established, the rest of the proof proceeds as in the proof of Theorem 1 .

6. It has been shown by Valiron" that if a function $f(z)$ is of class $(V)$ relative to a spiral, then, setting $M(r)=\max _{i=\mid \leqq r}|f(z)|$, we have

$$
\lim _{r \rightarrow 1} \frac{\log _{3} M(r)}{\log _{1-r} \frac{1}{1-r}} \geqslant 1
$$

In particular, this means that $f(z)$ must be of infinite order in $|z|<1$. On the other hand, the maximum modulus $M(r)$ of a function $f(z)$ which tends to infinity along a spiral path can be made to increase to infinity arbitrarily slowly.

Let $\omega(r)$ be any real, positive, strictly increasing function in $0 \leqq r<1$, such that $\lim _{r \rightarrow 1} \omega(r)=\infty$. Then, there exists a sequence of positive integers $\left\{n_{k}\right\}$ satisfying the conditions

$$
n_{1}>1, n_{k} \geqslant k n_{k-1} \text { for } k>1 \text {, }
$$

6. Valiron 2., loc. cit., pp. 13.3-43.5. 
such that the function ${ }^{7}$

$$
\Psi(z)=\prod_{j=1}^{\infty}\left\{1-\left(\frac{z}{1-n_{j}^{-1}}\right)^{n_{j}}\right\}
$$

is holomorphic in $|z|<1$ and, setting $M(r)=\max _{|z| \leqq r}|\Psi(z)|$, we have

$$
M(r)<\omega(r), 1-n_{1}^{-1} \leqq r<1 .
$$

Furthermore, if we denote by $\Omega$ the region formed by deleting from $|z|<1$ closed circular discs of radius $\frac{1}{j^{2}} \frac{1}{n_{j}}$ about the zeros of $\Psi(z),\left(1-n_{j}^{-1}\right) e^{2 \pi k i / n_{j}}$, $k=0,1,2, \ldots, n_{j}-1 ; j=j_{0}, j_{0}+1, \ldots$, (choosing $j_{0}$ sufficiently large so that the discs are mutually disjunct), then $|\Psi(z)|$ tends uniformly to infinity as $|z| \rightarrow 1$, with $z \in \Omega$.

Now, if we draw the two circumferences $|z|=1-\frac{1}{n_{j}} \pm \frac{1}{j^{2} n_{j}}$ on either side of $|z|=1-\frac{1}{n_{j}}$, we find that the $n-E$ distance between them is equal to

$$
\frac{1}{2} \log \left\{\frac{2 j^{2} n_{j}-j^{2}+1}{j^{2}-1} \cdot \frac{j^{2}+1}{2 j^{2} n_{j}-j^{2}-1}\right\}
$$

which tends to 0 as $j \rightarrow \infty$. Hence, it is clearly possible to construct a spiral $S$ wholly within the region $\Omega$ which satisfies the condition $\bar{\mu}(S)=0$. This shows that $\Psi(z)$ satisfies the condition of Theorem 3 . We thus obtain

Corollary 2. There exist functions, holomorphic in $|z|<1$, whose maximum modulus tends to infinity arbitrarily slowly, for which the conclusion of Theorem 3 holds.

7. Before continuing the study of functions with spiral asymptotic paths, we shall first prove a preliminary result of a more general character.

THEOREM 4. Let $f(z)$ be holomorphic in $|z|<1$, let $\tau$ be a point of $|z|=1$, and let $z_{n}=r_{n} \tau, 0<r_{n}<1, \lim _{n \rightarrow \infty} r_{n}=1$, be a sequence of points for which

$$
\lim _{n \rightarrow \infty} \rho\left(z_{n}, z_{n+1}\right)=0
$$

and

7) F. Bagemihl, P. Erdös, W. Seidel. Sur quelques propriétés frontières des fonctions holomorphes définies par certains produits dans le cercle-unité, Annales de l'École Normale Supérieure (3), vol. 70 (1953), pp. 135-147; in particular, pp. 136-141. 


$$
\lim _{n \rightarrow \infty}\left|f\left(z_{n}\right)\right|=\infty \text {. }
$$

Then, there exists a real number $\alpha_{-}$, with $0 \leqq \alpha_{:} \leqq \frac{\pi}{2}$, such that

1. $f(z)$ tends uniformly to infinity in every Stolz angle $A_{i, 3}$, where $\beta<\alpha_{\tau}$;

2. $C R\left(f, \Delta_{:, 3}\right)$ consists of at most one point for every Stolz angle $A_{:, 3}$, where $\beta>\alpha=.^{s)}$

Proof. For each value of $r$ in $0<r<1$ consider the function

$$
g_{r}(z)=f\left(\frac{z+r \tau}{1+r \tau z}\right)
$$

holomorphic in $|z|<1$. We have

$$
g_{r_{n}}(0)=f\left(r_{n} \tau\right)=f\left(z_{n}\right)
$$

so that, by (18), we obtain

$$
\lim _{n \rightarrow \infty}\left|g_{r_{n}}(0)\right|=\infty
$$

We shall now examine the family $\left\{g_{r}(z)\right\}$ for normality. There are altogether three mutually exclusive cases to be considered:

I. The family $\left\{g_{l}(z)\right\}$ is normal in $|z|<1$;

II. The family $\left\{g_{r}(z)\right\}$ is not normal in $|z|<1$, but is normal at $z=0$;

III. The family $\left\{g_{r}(z)\right\}$ is not normal at $z=0$.

Consider Case I. The subfamily $\left\{g_{r_{i}}(z)\right\}$ is also normal in $|z|<1$ and (19) implies that

$$
\lim _{n \rightarrow \infty}\left|g_{r_{n}}(z)\right|=\infty
$$

uniformly on every $\operatorname{disc}|z| \leqq q<1$. This, in turn, implies that $f(z)$ tends uniformly to infinity on the sequence of closed $n-E$ circular discs $D_{n}$ with $n-E$ centers $z_{n}$ and $n-E$ radius $\frac{1}{2} \log \frac{1+q}{1-q}$. Since, in view of (17), for $n$ sufficiently large, each $D_{n}$ contains in its interior the point $z_{n+1}$, the union $\bigcup_{n=1}^{\infty} D_{n}$

3) The extreme cases $x_{\tau}=0, \bar{\pi}$ must be interpreted to mean that, in the first case, conclusion 2 holds for every Stolz angle $A_{-, 3}$, while, in the second case, conclusion 1 holds for every Stolz angle $4:, 3$. 
of these discs contains some segment $0<\sigma<r<1$ of the radius $r \tau$ which terminates in $:$.

Hence,

$$
\lim _{r \rightarrow 1}|f(r \tau)|=\infty .
$$

Returning now to our original family $\left\{g_{r}(z)\right\}$, (20) implies that

$$
\lim _{r \rightarrow 1}\left|g_{r}(0)\right|=\infty \text {. }
$$

Making use of $I$ and the relation (21), by an argument similar to that employed in $\$ 5$, we infer that

$$
\lim _{r \rightarrow 1}\left|g_{r}(z)\right|=\infty
$$

uniformly on every $\operatorname{disc}|z| \leqq \lambda<1$. Applied to $f(z)$, this means that $f(z)$ tends uniformly to infinity as $|z| \rightarrow 1$ within the union of all $n-E$ closed circular discs $D_{r}$ with $n-E$ centers at $r$ and fixed $n-E$ radius $\frac{1}{2} \log \frac{1+\lambda}{1-\lambda}$. The union $\bigcup_{0<r<1} D_{r}$ fills out precisely the intersection of some neighborhood of $\tau$ with the region $H_{\tau, a}$, defined in $\S 4$, provided that

$$
\cot \left(\frac{\pi}{4}-\frac{\alpha}{2}\right)=\frac{1+\lambda}{1-\lambda}
$$

As $\lambda$ varies over the interval $0<\lambda<1$, the angle $\alpha$ varies over the interval $0<\alpha<\frac{\pi}{2}$. Since, in some neighborhood of $\tau$, each $H_{\tau, \alpha}$ contains every $\Delta_{\tau, \beta}$, with $\beta<\alpha$, we arrive at the conclusion that in Case I the point $\tau$ is a Fatou ${ }^{9)}$ point of $f(z)$ with the limit $\infty$, and we have $\alpha_{\tau}=\frac{\pi}{2}$.

Let us next consider Case III. In this case, the family $\left\{g_{r}(z)\right\}$ fails to be normal in every neighborhood $|z|<\lambda<1$ of $z=0$. Since for every interval $0<r \leqq b<1$, the subfamily $\left\{g_{r}(z), 0<r \leqq b<1\right\}$ is bounded in $|z|<\lambda$, hence normal, there must exist a sequence $\left\{g_{r_{u}}(z)\right\}$, with $r_{n} \rightarrow 1$, such that every value, except perhaps one, is assumed in $|z|<\lambda$ by infinitely many functions of the sequence. In terms of $f(z)$, this implies that $C R\left(f, H_{i, \alpha}\right)$ consists of at most one point for every domain $H_{i, \alpha}$. This is equivalent to asserting that in

9) A point $\tau$, with $|\tau|=1$, is called a Fatou point of a function $f(z)$, meromorphic in $|z|<1$, if there exists a number $c$, finite or infinite, such that $f(z) \rightarrow c$ as $z \rightarrow \tau$ uniformly in every Stolz angle $\Delta_{\tau}$. . Cf. Collingwood and Cartwright, loc. cit., p. 95. 
Case III, CR $\left(f, \Delta_{\tau, \alpha}\right)$ consists of at most one point for every Stolz angle $\Delta_{\tau, \alpha}$, and we have $\alpha_{\tau}=0$.

Finally, in Case II, let $0<\sigma<1$ be the smallest modulus of all those points in $|z|<1$ at which $\left\{g_{r}(z)\right\}$ fails to be normal. Since the set of such points is closed relative to $|z|<1$, such a smallest positive modulus exists. Let $\alpha_{\tau}\left(0<\alpha_{\tau}<\frac{\pi}{2}\right)$ be that angle $\alpha$ for which

$$
\cot \left(\frac{\pi}{4}-\frac{\alpha}{2}\right)=\frac{1+\sigma}{1-\sigma}
$$

Then, $f(z)$ will tend uniformly to infinity as $z \rightarrow+$ in every Stolz angle $\Delta_{:, \beta}$, with $\beta<\alpha_{i}$, and $C R\left(f, \Delta_{\beta, \alpha}\right)$ will consist of at most one point for $\beta>\alpha_{i}$.

This completes the proof of Theorem 4.

We list some immediate corollaries of Theorem 4.

Corollary 3. Let $f(z)$ be holomorphic in $|z|<1$ and omit ${ }^{10)}$ two distinct finite values there. Let $\tau$ be a point of $|z|=1$ and $z_{n}=r_{n} \tau, 0<r_{n}<1, \lim _{n \rightarrow \infty} r_{n}=1$, a sequence of points for which

$$
\lim _{n \rightarrow \infty} \rho\left(z_{n}, z_{n+1}\right)=0
$$

and

$$
\lim _{n \rightarrow \infty}\left|f\left(z_{n}\right)\right|=\infty
$$

Then $\tau$ is a Fatou point of $f(z)$ with the limit $\infty$.

Since, under these assumptions, conclusion 2 of Theorem 4 can not take place, we have $\alpha_{\tau}=\frac{\pi}{2}$.

It is obvious what assumptions are needed to ensure that conclusion 1 will not take place, so that $\alpha_{\tau}=0$, and conclusion 2 holds in every Stolz angle $\Delta_{:, \alpha}$.

Corollary 4. Let $f(z)$ be meromorphic in $|z|<1$ and omit three distinct values $a, b, c$, finite or infinite, there. Let $\tau$ be a point of $|z|=1$ and $z_{n}=r_{n} \tau$, $0<r_{n}<1, \lim _{n \rightarrow \infty} r_{n}=1$, a sequence of points for which (22) holds and

10) It clearly suffices to assume here that $f(z)$ assumes two distinct finite values at most a finite number of times. An analogous extension of Corollary 4 is also evidently true. 


$$
\lim _{n \rightarrow \infty} f\left(z_{n}\right)=a
$$

Then $\tau$ is a Fatou point of $f(z)$ with the limit $a$.

The case $a=\infty$ is precisely Corollary 3. If $a \neq \infty$, Corollary 3 applied to the function $\frac{1}{f(z)-a}$ immediately yields a proof.

Corollary 5. Let $f(z)$ be holomorphic in $|z|<1$. Let $\tau$ be a point of $|z|=1$ for which

$$
\lim _{r \rightarrow 1}|f(r \tau)|=\infty
$$

Then, there exists a number $\alpha_{-}$, with $0 \leqq \alpha_{-} \leqq \frac{\pi}{2}$, for which the conclusion of Theorem 4 holds.

This is merely a special case of a theorem proved by Gross. ${ }^{11}$

Another immediate consequence of Theorem 4 is

TheOREM 5. Let $f(z)$ be holomorphic in $|z|<1$ and let $S: z=\zeta(t), 0 \leqq t<\infty$, be a spiral with $\bar{\mu}(S)=0$, such that $\lim _{t \rightarrow \infty}|f(\zeta(t))|=\infty$. Then, to every point $\tau$ of $|z|=1$ one can assign a number $\alpha_{-}$, with $0 \leqq \alpha_{i} \leqq \frac{\pi}{2}$, so that the conclusion of Theorem 4 holds.

8. We return to the function $\Psi(z)$ defined by (16) in $\S 6$. Since, as pointed out there, it is possible to draw a spiral $S$, with $\bar{\mu}(S)=0$, on which $\Psi(z)$ tends to infinity, the function $\Psi(z)$ satisfies the conditions of Theorem 5 , so that we can associate with every $\tau,|\tau|=1$, a number $\alpha_{\tau}$. We shall show that in this case there exists a fixed number $\alpha_{0}$, with $0<\alpha_{0}<\frac{\pi}{2}$, such that for every $\tau$, $|\tau|=1, C R\left(\Psi, \Delta_{\tau, \beta}\right)$ consists of at most one point whenever $\beta>\alpha_{0}$.

We shall show, in fact, that every Stolz angle $\Delta_{t, \beta}$ for $\beta>\alpha_{0}$ contains infinitely many zeros of $\Psi(z)$, which suffices to establish the assertion. It is clear, first of all, that every arc of the circle $|z|=1-\frac{1}{n_{k}}$, of length greater than $\frac{2 \pi}{n_{k}}\left(1-\frac{1}{n_{k}}\right)$ must contain at least one zero of $\Psi(z)$. Let us select any

11) W. Gross. Über die Singularitäten analytischer Finktionen, Monatshefte für Mathematik und Physik, vol. 29 (1918), pp. 3-47; particularly, p. 26. 
point $\tau$ on $|z|=1$ and the Stol $z$ angle $\Delta_{\tau, \beta}$, where $0<\beta<\frac{\pi}{2}$. If $c_{k}$ denotes the length of the chord which subtends the smaller arc $\sigma_{k}$ intercepted by $A_{:, \beta}$ on the circle $|z|=1-\frac{1}{n_{k}}$, an elementary calculation shows that $\lim _{k \rightarrow \infty}\left(n_{k} \cdot c_{k}\right)=$ $2 \tan \beta$. Hence, for $k$ sufficiently large, the arc $\sigma_{k}$ will be greater than $\frac{2 \pi}{n_{k}}\left(1-\frac{1}{n_{k}}\right)$ provided that $\beta>\alpha_{0}$, where $\alpha_{0}$ is determined by the equation $\tan \alpha_{0}=\pi$.

Since, at the same time, $\Psi(z)$ can be chosen so that its maximum modulus tends to infinity arbitrarily slowly, we have

THEOREM 6. There exists a function $\Psi(z)$, holomorphic in $|z|<1$, whose maximum modulus tends to infinity as slowly as one wishes, with the property that there exists a fixed number $\alpha_{0}$, with $0<\alpha_{0}<\frac{\pi}{2}$, such that $C R\left(\Psi, \Delta_{\tau, \beta}\right)$ consists of at most one point for every $\tau,|\tau|=1$, and every $\beta>\alpha_{0}$.

Since we have $0<\alpha_{0}<\frac{\pi}{2}$ in the preceding theorem, we see that no point of the circumference $|z|=1$ can be a Fatou point. Applying now a theorem due to $A$. Plessner, ${ }^{12)}$ we infer that at almost all points $\tau$ of $|z|=1$, the cluster set $^{13)}$ of $\Psi(z)$ at $\tau$ within any Stolz angle with vertex $\tau$ is the whole Riemann sphere. In view of Theorem 4, this implies in turn that for almost all points $\tau$ of $|z|=1, \alpha==0$, whence we infer

THEOREM 7. There exists a function ${ }^{14)} \Psi(z)$, holomorphic in $|z|<1$, whose maximum moaulus tends to infinity as slowly as one wishes, with the property that at almost all points $\tau$ of $|z|=1, C R(\Psi, \Delta \tau, \alpha)$ consists of at most one point for every Stolz angle $\Delta_{\tau, \alpha}$.

University of Notre Dame

12) A. Plessner. Über das Verhalten analytischer Funktionen am Rande ihres Definitions. bereiches, Journal für die reine und angewandte Mathematik, vol. 158 (1927), pp. 219-227. 139.

13) For the definition of this term, see e.g. Collingwood and Cartwright, loc. cit., p.

14) It is to be noted that both Theorems 6 and 7 are valid for the same function. 